Research Article

\title{
The Liquid Maldistribution Analysis of the Trickle Bed Reactor with the CFD Method
}

\author{
Jinjin Liu $\mathbb{D}^{\mathbb{D}}$, Liu Kai, Tong Zhao $\mathbb{D}$, and Chuanxin Bai \\ Mechanical and Precision Instrument Engineering, Xi'an University of Technology, Xi'an 710048, China \\ Correspondence should be addressed to Tong Zhao; tongzhao@xaut.edu.cn
}

Received 9 April 2020; Revised 14 June 2020; Accepted 8 July 2020; Published 5 August 2020

Guest Editor: Wenqing Wang

Copyright (c) 2020 Jinjin Liu et al. This is an open access article distributed under the Creative Commons Attribution License, which permits unrestricted use, distribution, and reproduction in any medium, provided the original work is properly cited.

\begin{abstract}
The liquid phase maldistribution factor has been investigated in trickle bed reactor, and the results are compared with the previous measurement data from literature by using the Electrical Resistance Tomography. The simulation results are in agreement with the experimental results to some degree. The flow rates and particle sizes have been simulated with the method of multiphase flow. There are two different particles with average diameters of $3.4 \mathrm{~mm}$ and $5.3 \mathrm{~mm}$. The flow rate has been studied ranging from $100 \mathrm{ml} / \mathrm{min}$ to $1100 \mathrm{ml} / \mathrm{min}$. It has been found that the changes of the particles and liquid flow rates have a significant impact on the distribution of the liquid volume fraction. The internal liquid holdup is more serious, and the wall-flow phenomenon is more obvious in a bigger flow rate. The prediction of the liquid volume fraction distribution is a key research technique. Regression predictions have also been researched on the section near outlet, which can predict the internal flow state of the trickle bed under the condition of high temperature and high pressure. The average liquid volume fraction is linear with flow rates. The maldistribution factor is the index correlation with the flow rates. The results and main conclusions can be used to predict the distributions and get the properties in a trickle bed reactor.
\end{abstract}

\section{Introduction}

The trickle bed reactor has been applied in various fields, such as petrochemical, environmental protection, and energy conversion [1]. There are three phases in the trikle bed reactor which are contacted with each other, and the gas and liquid pass together through the solid phase, which stands for the catalyst particles at work [2]. Trickle bed reactors play an important role in most catalytic reactions in various industries. Since the efficiency and production in catalytic reaction are always decided by the distribution and velocity of gas and liquid phases, it is useful to get the properties of multiphase phases in TBR to evaluate and control the reaction. However, it is hard to obtain the status of internal flow in a TBR, and that is because TBR cannot be observed outside directly. Detection with normal sensors is also not easy due to its high temperature and pressure inside [3].

To solve the problem mentioned above, CFD methods are usually chosen by many experts. Bazmi et al. [4] investigated a numerical model for liquid maldistribution in random trickle bed reactors. A model based on the threephase Eulerian approach is developed and a two-fluid model is utilized to perform the interphase momentum exchanges. Then, Beni and Khosravi [5] made a research on the pressure drop and liquid holdup in high- and low-pressure tricklebed reactors based on CFD. Rupesh and Jyeshtharaj [6] have investigated the effect of the trickle bed reactor on particle diameter ratio and verified that the predicted values of drag coefficient are consistent with the experimental results in the literature. Mohammad et al. [7] presented a combination of CFD and Adaptive Network-based Fuzzy Inference System developed for flow characterization inside a cylindrical bubble column reactor. And the liquid flow pattern and gas dynamics were predicted. Amir and Faical [8] have used a Euler-Euler porous media CFD model to elucidate the dynamic properties of gas-liquid flows with moving frame and sliding mesh techniques. And from these works, it can be seen that CFD is an effective method and used in TBRs.

In order to get a real distribution in TBRs, some medical imaging techniques have been applied to make visualization 
for liquid distribution, such as x-ray computed tomography, gamma-ray CT, and magnetic resonance imaging (MRI) method. X-ray computed tomography have been used in a research on two-phase flow in two different types of squarechannel monolith structures by Schafer et al. [9]. The gamma-ray tomography was used to study the distribution and flow rate of liquid and gas phases in a trickle bed by Christophe et al. [10]. Gladden et al. [11] took MRI to measure the liquid holdup and the wetting efficiency in two different reactor environments. Fuhe et al. [12] have measured the spatial liquid holdup in TBRs also by the gammaray tomography, and the result of CFD simulation agreed with the experimental one. Wang et al. [13] have detected the gas-liquid pulsating flows in the trickle bed reactor with the electrical capacitance volume tomography system. These measurement techniques are useful in some situation but always have a low temporal resolution and very high cost. These complex setups are hard to monitor or use in daily work. Therefore, ERT is getting more attention in TBR due to its convenience and receptive price. Mcmanus et al. [14] have investigated the distribution of only a single liquid phase under different flow rates in TBR by ERT. Then, Eda et al. [15] have investigated the gas-liquid flow distribution of spherical particles in the packed bed by ERT and found that the liquid flow rate has an influence on the radial diffusion of liquid phase.

However, the experimental measurement of the amount for liquid distribution has a time lag. Simultaneously, the environmental and operational errors have a great influence on results. The internal working conditions of the trickle bed reactor are always under high temperatures and pressures, and it is difficult to reveal the distribution of liquid phase in time. In order to overcome the difficulties mentioned above, a simulation for macroscopic multiphase phase flow of gasliquid in TBR is studied based on CFD, and the results have also been compared with the experiment of ERT in [16].

The contents of this paper are as follows. Firstly, a CFD model is established, and its boundary conditions are set according to an ERT work. Secondly, the simulation results, with two different particle sizes and three different liquid flow rates, have been obtained. The maldistributions of the liquid phase are compared with the experiment. Furthermore, regression models have been taken and the effect on the distribution of the liquid phase from different flow rates and particle diameters is also analyzed. Finally, the model can establish a relationship between the maldistribution of liquid volume fraction and various flow rates.

\section{Method}

2.1. The Theory of the Numerical Simulation. The simulation method in TBR mainly consists of two steps. Firstly, a distribution with proper porosity should be set in the trickle bed reactor, and the distribution of porosity in simulation should be consistent with the measured in experiment. Then, a mixture model of multiphase flow is built to simulate the gas-liquid two-phase flow.

The particles with an average diameter of $3.4 \mathrm{~mm}$ have a porosity of 0.408 and a deviation of 0.3 , while those with an average diameter of $5.3 \mathrm{~mm}$ have a porosity of 0.431 and a deviation of 0.3 , and conditions not given are the same as [17]. The shape of the particles is spherical with uniform diameters. Besides, the permeability and the coefficient of inertial principal force need to be chosen in later CFD computations, and they can be calculated as follows:

$$
\begin{gathered}
\frac{1}{\alpha}=\frac{150}{D^{2}} \frac{(1-\varepsilon)^{2}}{\varepsilon^{3}}, \\
C=\frac{3.5}{D} \frac{(1-\varepsilon)}{\varepsilon^{3}},
\end{gathered}
$$

where $D$ is the diameter of particles here, $\varepsilon$ means the porosity, $\alpha$ means the permeability, and $C$ is the inertia loss coefficient.

A mixture model of multiphase flow is used in CFD. As to the mixture model, changing of gas and liquid is the main factors, since the catalytic agent is always in a relatively static position and its moving can be neglected here. Then, the gas and liquid are set as continuous phases. The mass and momentum volume mean conservation equations for gasliquid phases are solved. The application of conservation equations for the mass and momentum is done by averaging the summing of the local instantaneous equilibriums in each phase [18]. In general condition of TBR, the gas phase and liquid phase can be considered as the incompressible fluids. Then, the continuity equation can be presented as follows:

$$
\frac{\partial}{\partial t}\left(\rho_{m}\right)+\nabla \cdot\left(\rho_{m} \vec{v}_{m}\right)=0
$$

Here, $\overrightarrow{v_{m}}$ is the averaged velocity of mass and expressed as equation (4). $\rho_{m}$ means the mixture density and can be expressed as equation (5). $\alpha_{k}$ is the volume fraction of phase $k$.

$$
\begin{aligned}
& \vec{v}_{m}=\frac{\sum_{k=1}^{n} \alpha_{k} \rho_{k} \vec{v}_{k}}{\rho_{m}}, \\
& \rho_{m}=\sum_{k=1}^{n} \alpha_{k} \rho_{k} .
\end{aligned}
$$

The momentum conservation equation can be derived by summing the momentum equations of all phases. It can be expressed as equation (6).

$$
\begin{aligned}
\frac{\partial}{\partial t}\left(\rho_{m} \vec{v}_{m}\right)+\nabla \cdot\left(\rho_{m} \vec{v}_{m} \vec{v}_{m}\right)=-\nabla p & +\nabla \cdot\left[\mu_{m}\left(\nabla \vec{v}_{m}+\nabla_{\vec{v}_{m}}^{T}\right)\right]+\rho_{m} \vec{g} \\
& +\vec{F}+\nabla \cdot\left(\sum_{k=1}^{n} \alpha_{k} \rho_{k} \vec{v}_{d r, k} \vec{v}_{d r, k}\right),
\end{aligned}
$$

$$
\begin{aligned}
\mu_{m} & =\sum_{k=1}^{n} \alpha_{k} \mu_{k}, \\
\vec{v}_{d r, k} & =\vec{v}_{k}-\vec{v}_{m} .
\end{aligned}
$$

Here, $n$ is the number of phases and $\vec{F}$ is a body force. $\mu_{m}$ is the viscosity of the phases. The relationship between the drag force and mass transfer can be treated as closed terms to 
calculate the flow state of each phase in the multiphase flow and written as follows [19]:

$$
\begin{gathered}
F_{g l}=\frac{E_{1} \mu_{g} \varepsilon_{g}\left(1-\varepsilon_{g}\right)^{2}}{\varepsilon_{g}^{2} d_{p}^{2}}\left[\frac{\varepsilon_{s}}{1-\varepsilon_{g}}\right]^{2 / 3} \\
+\frac{E_{2} \rho_{g} \varepsilon_{g}\left(1-\varepsilon_{g}\right)\left(u_{g}-u_{l}\right)}{\varepsilon_{g} d_{p}}\left[\frac{\varepsilon_{s}}{1-\varepsilon_{g}}\right]^{1 / 3}, \\
F_{g s}=\frac{E_{1} \mu_{g} \varepsilon_{s}\left(1-\varepsilon_{g}\right)^{2}}{\varepsilon_{g}^{2} d_{p}^{2}}\left[\frac{\varepsilon_{s}}{1-\varepsilon_{g}}\right]^{2 / 3}+\frac{E_{2} \rho_{g} \varepsilon_{s}\left(1-\varepsilon_{g}\right)}{\varepsilon_{g} d_{p}}\left[\frac{\varepsilon_{s}}{1-\varepsilon_{g}}\right]^{1 / 3}, \\
F_{l s}=\varepsilon_{1}\left(\frac{E_{1} \mu_{l} \varepsilon_{s}^{2}}{\varepsilon_{l}^{2} d_{p}^{2}}+\frac{E_{2} \rho_{l} \mu_{g} \varepsilon_{s}}{\varepsilon_{l} d_{p}}\right)
\end{gathered}
$$

where $E_{1}$ and $E_{2}$ are Ergun constants [19]. $E_{1}$ is set to 1.5 and $E_{2}$ is 1.75 in a normal simulation by experience. $\rho_{l}$ and $\rho_{g}$ are the liquid density and the gas density, respectively. $u_{l}$ and $u_{g}$ are the velocity of liquid and gas, respectively. $d_{p}$ is the diameter of the porous medium region. $\varepsilon_{l}$ and $\varepsilon_{g}$ are the liquid phase holdup and gas phase holdup, respectively. $\varepsilon_{s}$ means the volume fraction of solid.

\subsection{Model and Condition Settings}

2.2.1. Simulation Model. The CFD model has the same size as the experimental apparatus as shown in Figure 1(a). Four sections are chosen for analysis. The symbols of P1, P2, P3, and $\mathrm{P} 4$ present the different sections with a distance of $60 \mathrm{~mm}, 180 \mathrm{~mm}, 300 \mathrm{~mm}$, and $420 \mathrm{~mm}$, respectively, from the bottom of TBR. The inlet is on the top, and there are 9 round holes with a diameter of $3 \mathrm{~mm}$. The middle round hole is the inlet of liquid and the other 8 round holes are inlets of air around it. The relationship and sizes between inlets are described in Figure 1(b).

The mesh node spacing of the area between P1 and P4 is set as 10, and both inlets and outlets parts are set as 3 with a more precise result. Constrained by computing power, it is difficult to set all meshes with a spacing of 3 . The total number of meshes is 280536. Considering the volume of whole model, the mesh size is smaller than a particle with a diameter of $3.4 \mathrm{~mm}$ or $5.3 \mathrm{~mm}$. A workstation is used to simulate the model: the CPU is Inter Xeon E3-1231 v3 and computing memory is $16 \mathrm{G}$. The operating system is Windows 10. The hexahedral mesh is used in the entire model. The convergence of calculation is determined by the residual of the mass continuity equation steadied less than $10^{-6}$.

2.2.2. Simulation Setup. General information on the simulation setups is presented in Table 1. The simulation is based on the unsteady state solver of pressure-continuous with FLUENT. The discretization scheme of second order is also used on the momentum and phase fractions. The liquid phase is injected in the fluid zone from the inlet. The particle filling area is treated as the porous medium which is laminar flow model, and the rest is set to a turbulent model in this work. The bottom is treated as the exit, which is free flow model. The gas is fed into the porous medium to the packing uniformly. Some boundary conditions of simulation not mentioned here are set according to the experiments in [16]. Viscous resistance and inertial resistance are defined according to the result of equations (1) and (2). Face permeability reflects the state of being permeable in the section, while porous permeability reflects the ratio of permeable volume to whole immersed volume in porous medium.

Multiphase flows in trickle-beds are often characterized by low interaction regimes so that the flow is often assumed laminar either at the reactor level or at the catalyst particle scale [20]. Therefore, laminar flow model is chosen to study the multiphase of spaces between particles in some works [21]. Considering that the situation in inlets and outlets is more complex, turbulent model is also used in this work, as a supplement, besides the laminar flow mode.

2.2.3. The Experiment Setup of Electrical Resistance Tomography. The Electrical Resistance Tomography is one of the electrical tomographic techniques; the cross-sectional electrical conductivity distribution can be obtained with ERT. The ERT setup consists of four sensors, a data acquisition system, and related image reconstruction software. In ERT, an array of electrodes is arranged on the border of the trickle reactor to obtain a sensitive field of the object field in different directions. The electrodes are measured to obtain projection data in the different scanning directions. Then, the conductivity distribution in the porous media field is estimated from the vector of projection data using an image reconstruction algorithm to realize the visualization of the liquid distribution in the object field. The adjacent measurement strategy is used here, which is to inject current between two adjacent electrodes and measure the voltage of all other two adjacent electrode pairs. The pairs of injecting current are moved to next adjacent pair and voltage measurements are repeated [22].

The distributions of inlets, sections, and outlet are nearly the same in Figure 1. The positions of data obtained are shown in Figure 2. The height of the trickle bed is $600 \mathrm{~mm}$. The particle packing part of the trickle reactor has a length of $480 \mathrm{~mm}$ and an inner diameter of $100 \mathrm{~mm}$. The experimental setup and its structure sizes are recorded in [16]. The spherical activated alumina particles are used for catalyst support in the reactor, and the average diameters of packing particles are $3.4 \mathrm{~mm}$ and $5.3 \mathrm{~mm}$, respectively. Water was used as liquid. In order to provide enough conductivity for detection, $\mathrm{NaCl}$ of $0.5 \%$ was mixed in water. The conductivity value is $10 \mathrm{mS} / \mathrm{cm}$. The data acquisition system has an inject current of $50 \mathrm{~mA}$ and an excitation frequency of $9600 \mathrm{~Hz}$. The cross-sectional images were acquired every 10 seconds at each of the four sensors.

\section{Results}

In this paper, the liquid phase volume fraction in the TRB is treated as the research parameter. Four sections are selected and represented by the symbol P1, P2, P3, and P4, 


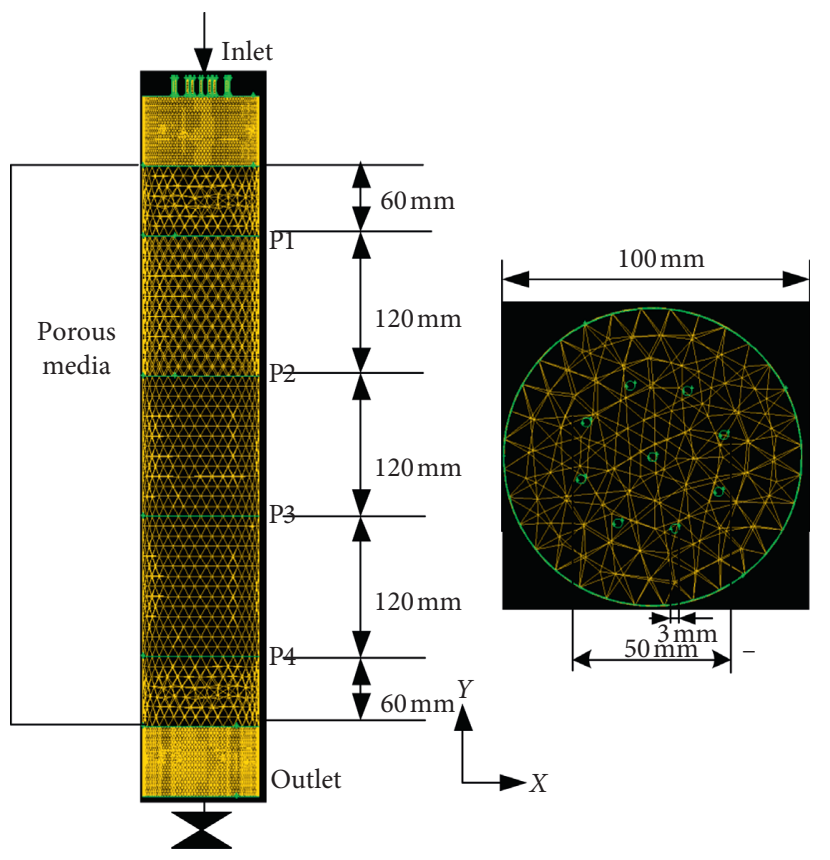

(a)

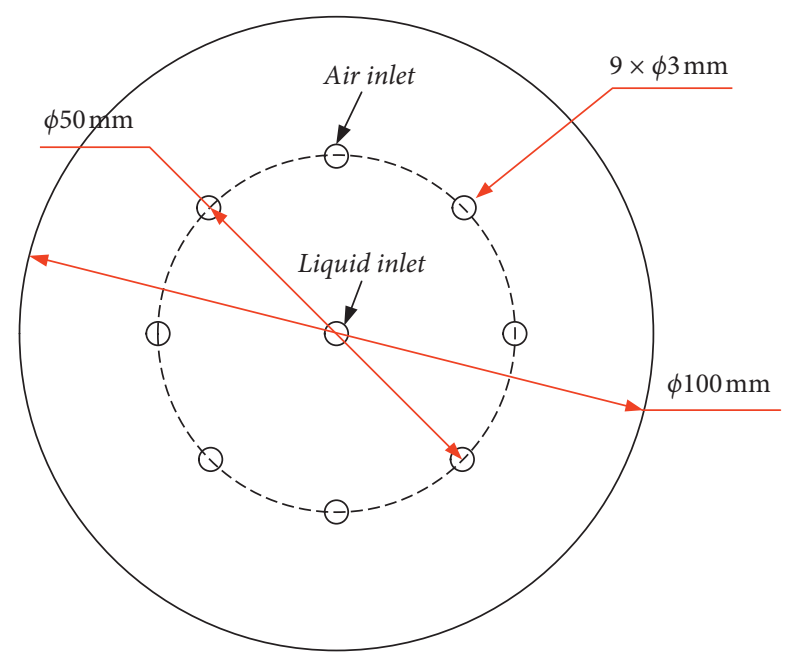

(b)

FIgure 1: The 3D simulation model. (a) Meshed model. (b) Distribution of inlets (mm).

TABLE 1: The parameter values of the multiphase flow and porous area.

\begin{tabular}{|c|c|c|}
\hline \multirow{12}{*}{ Simulation setup } & Gas phase density $\left(\mathrm{kg} / \mathrm{m}^{3}\right)$ & 1.225 \\
\hline & Gas phase viscosity $\left(\mathrm{kg} / \mathrm{m}^{-s}\right)$ & $1.7894 e^{-5}$ \\
\hline & Reference temperature $(\mathrm{k})$ & 298.15 \\
\hline & Turbulent kinetic energy & 10 \\
\hline & Turbulent dissipation rate & 0.4 \\
\hline & Liquid phase density $\left(\mathrm{kg} / \mathrm{m}^{3}\right)$ & 998.2 \\
\hline & Liquid phase viscosity $\left(\mathrm{kg} / \mathrm{m}^{-\mathrm{s}}\right)$ & 0.001003 \\
\hline & Face permeability $\left(\mathrm{m}^{2}\right)$ & $1.4925 e^{-8}$ \\
\hline & Porous permeability & 0.5 \\
\hline & Viscous resistance & $6.7 \mathrm{e}^{+7}$ \\
\hline & Inertial resistance & $2.17 \mathrm{e}^{+3}$ \\
\hline & Porous medium thickness $(\mathrm{m})$ & 0.05 \\
\hline \multirow{5}{*}{ Experimental setup } & Cylindrical diameter $(\mathrm{mm})$ & 100 \\
\hline & Packing height $(\mathrm{mm})$ & 480 \\
\hline & Single point source inlet diameter $(\mathrm{mm})$ & 3 \\
\hline & Porosity & $0.408 / 0.431$ \\
\hline & Liquid flow rate $(\mathrm{ml} / \mathrm{min})$ & $100-1100$ \\
\hline
\end{tabular}

respectively. The position of four sections is $60 \mathrm{~mm}, 180 \mathrm{~mm}$, $300 \mathrm{~mm}$, and $420 \mathrm{~mm}$. In the experiment of ERT, 316 points are selected due to the test accuracy in experimental apparatus. In the CFD work, 108 points in a section are extracted. The numbers are different but all points distribute evenly. The maldistribution factor is introduced to evaluate quantitatively the uniformity distribution of liquid phase volume fraction. It is often used in similar problems. The maldistribution factor is the ratio of variance to mean, and the calculation formula is shown as follows:

$$
M_{f}=\frac{\sqrt{1 / n \sum_{i=1}^{n}\left(\bar{c}-c_{i}\right)^{2}}}{\bar{c}} .
$$

Here, $n$ is the number of selected points. 108 points were obtained from each section every 10 seconds, and the total time is 4 minutes. $\bar{c}$ represents the average value of the selected points. The average value is given by

$$
\bar{c}=\sqrt{\frac{1}{n} \sum_{i}^{n} c_{i}^{2}}
$$

where $c_{i}$ is the single value from the sample date. The value of $M_{f}$ will become zero when the values of the liquid phase volume fraction are the same in sections. The larger value of $M_{f}$ represents the uneven distribution of the liquid volume fraction. 


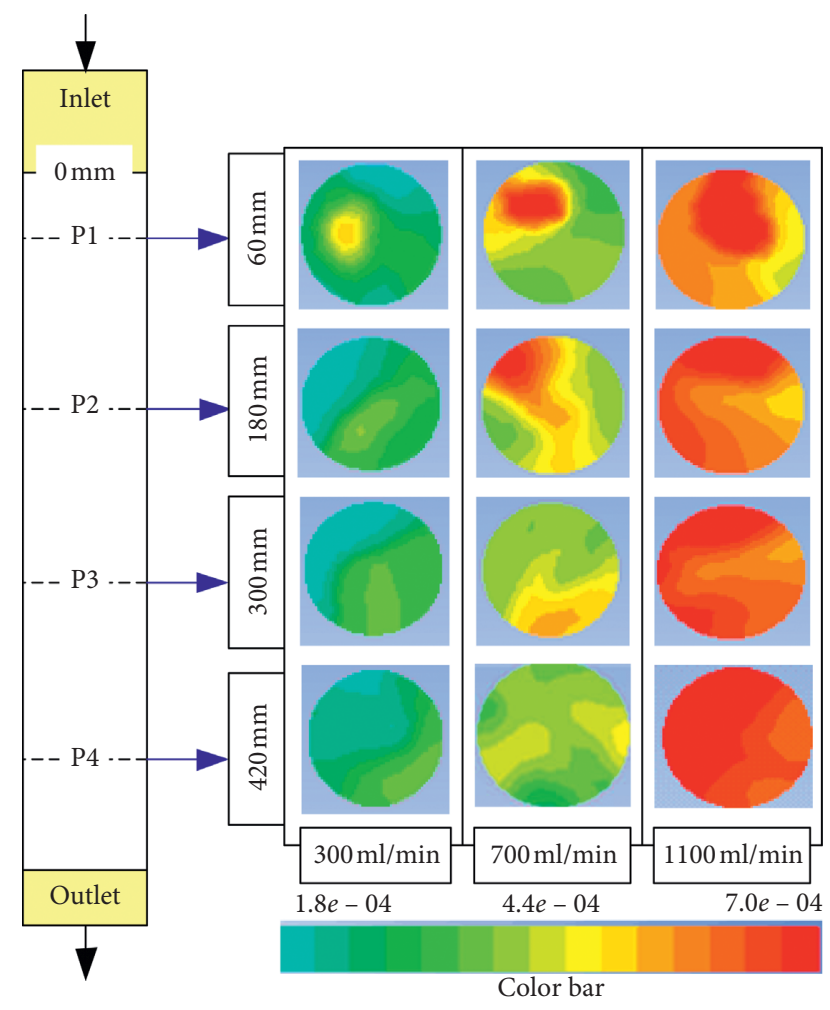

FIGURE 2: The image of liquid volume fraction in simulation with $5.3 \mathrm{~mm}$ particles.

3.1. The Analysis of Reconstruction Image. The visualization of those four sections in CFD models is taken after calculation and the result is shown in Figure 2. The reconstructed images of conductive are recorded in [16]. In this part, the particles with a diameter of $5.3 \mathrm{~mm}$ are studied under three flow rates including $300 \mathrm{ml} / \mathrm{min}, 700 \mathrm{ml} / \mathrm{min}$, and $1100 \mathrm{ml} /$ min. In order to avoid an over-discipline in calculation, the board condition in outlet is set to be free.

Figure 2 reflects the value of liquid phase volume fraction and most of the volume fraction is taken by particle phase. Then, there is a certain height between the inlets and sections; the gas phase takes a large part of volume fraction since it is easy to be trapped in the particle gap. The flow of liquid phase has also been resisted in a large degree due to the complexity of porous media penetration and the interaction between gas and liquid. Therefore, the volume fraction of liquid phase in the whole cross section is relatively small. Considering the reasons mentioned above and the obtained distribution of ERT in the following part, it is still reasonable and reflects the real properties of liquid phase to some degree.

Some common trends are found in both results of CFD and ERT. Firstly, with the increase of flow rate, the volume fraction grows in all sections obviously. The trends of average values and maldistributions are similar to a certain extent. Maldistributions are recorded in Figures 3 and 4 and it can be found that trends are similar. Although the values are different, they are still in an order of magnitude. A more specific discussion is in the next part.

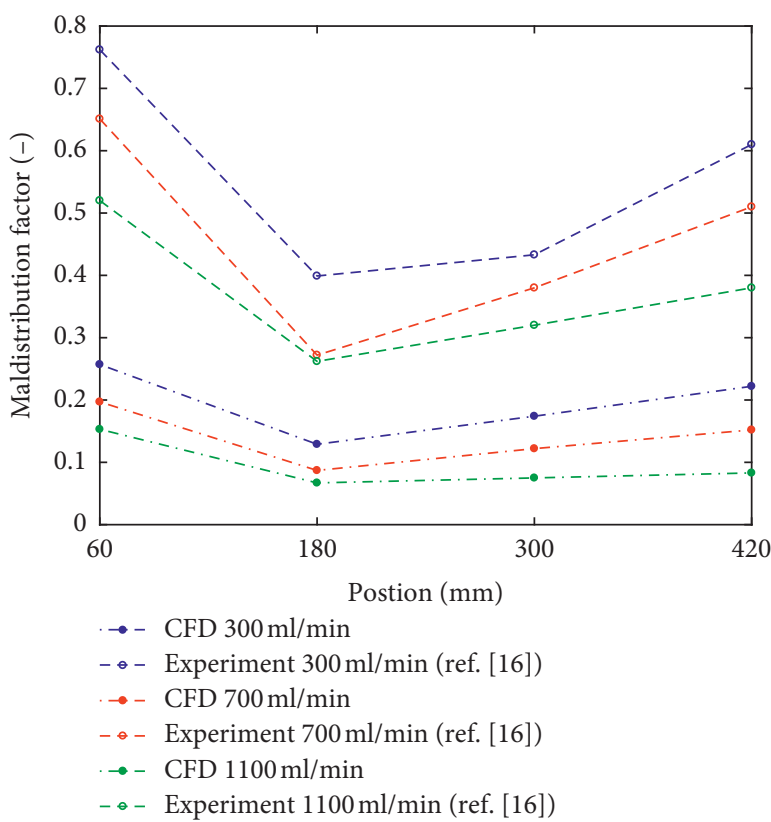

FIgURE 3: The maldistribution factor with $5.3 \mathrm{~mm}$ particles.

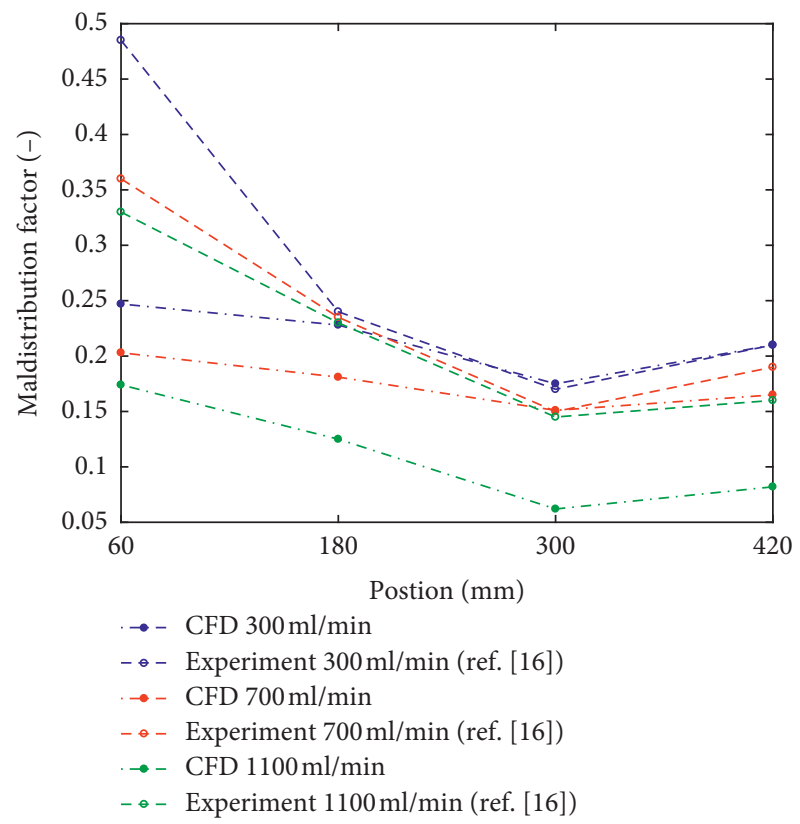

Figure 4: The maldistribution factor with $3.4 \mathrm{~mm}$ particles.

Meanwhile, wall-flow can be found in both CFD and ERT, and it will be more obvious with a higher flow rate. Comparing sections $\mathrm{P} 1, \mathrm{P} 2$, and $\mathrm{P} 3$, it can be seen that wallflow is more obvious near inlet, and a central region with a higher volume fraction, which reflects the position of inlet for liquid, can be found in them and it. In section P2, a deviation occurred in both kinds of images and the central region turns to wall. Then, the central area gradually spreads and a wall-flow happens in P3.

The wall-flow appears in the same section of CFD and ERT but the positions are quite different, and this is because 
it is hard to simulate the actual form of particles and the surface tension of liquids in CFD. Actually, resistance wires have to be used in ERT; thus the distribution will certainly be changed to some degree. Therefore, there is surely a difference from actual work. In addition, there is a more obvious deviation of P4 in the ERT and it tends to be more uniform later. This deviation is caused by the differences between real condition and simulation, and it only can be reduced but hard to eliminate completely.

3.2. The Distribution under Different Diameters. The distribution of particle has an influence on the volume fraction of liquids and thus two different particles, $3.4 \mathrm{~mm}$ and $5.3 \mathrm{~mm}$, are both studied in three different flow rates. Four sections are taken, and the maldistribution factors are shown in Figure 3. The results of the liquid phase distribution at the $3.4 \mathrm{~mm}$ particle diameter are presented in Figure 4.

From the figures, it can be seen that all the values of $5.3 \mathrm{~mm}$, in both CFD and ERT, are higher than $3.4 \mathrm{~mm}$. It can be concluded that liquid phase flow is prevented in a greater extent with a smaller diameter; still the trends of two samples are very similar to each other.

In order to compare the results from different fields, a dimensionless unit, maldistribution factor, has to be used. The degree of dispersion for distribution is described by the maldistribution factor. The experiment data in Figures 3 and 4 comes from [16]. From Figure 3, it can be seen that maldistribution factor declines at the beginning of P1 and then reaches a minimum near section P2. However, it increases after minimum value until it leaves the TBR. In Figure 4, the minimum value is near section P3 due to the difference of diameters. The process means that the liquid is relatively uneven near inlet and then is blocked by the particles. As the process continues, bigger pressure comes out between P2 and P3 and therefore causes the liquid to flow more slowly and uniformly. From this part, a clearer process can be observed and the effect of maldistribution is also proved. In the process, values of $5.3 \mathrm{~mm}$ particles reduce more quickly than the $3.4 \mathrm{~mm}$ particles; meanwhile, the steep rises happen with larger particles, and this phenomenon reflects that a wall-flow is more obvious with a larger diameter.
The average values are also compared between CFD and ERT. Since the principles in two methods are quite different, the units, volume fraction in CFD and conductivity in ERT, are far away from each other, and thus they cannot be compared directly. In order to resolve this problem, average values are normalized and then can be studied reasonably.

The original data in CFD is compared with the result in [16]. Calculate mean values from both CFD and ERT based on the min-max normalization. The min-max normalization is commonly used to get dimensionless parameters. It changes data into a range from 0 to 1 . The calculation can be described as equation (14). $x_{i}$ means a value to be calculated and $x_{i}^{\prime}$ means the normalized data. $x_{\max }^{\prime}$ and $x_{\min }^{\prime}$ were set as 1 and 0 before calculation.

$$
x_{i}^{\prime}=\left(x_{\max }^{\prime}-x_{\min }^{\prime}\right) \times \frac{x_{i}-x_{\min }}{x_{\max }-x_{\min }}+x_{\min } .
$$

Relative error between ERT and CFD is used to evaluate the uniformity. Since a true value cannot be obtained here, the denominator of relative error is replaced by a larger value between $x_{\mathrm{ERT}}$ and $x_{\mathrm{CFD}}$ as equation (15). Therefore, the relative error is still in a range from $0 \%$ to $100 \%$ and negative values will not appear. In addition, a value of 0 was added to the data before normalization to keep the minimum as 0 here.

$$
\text { error }=\left|\frac{x_{\mathrm{CFD}}-x_{\mathrm{ERT}}}{\operatorname{Max}\left\{x_{\mathrm{CFD}}, x_{\mathrm{ERT}}\right\}}\right| \times 100 \% \text {. }
$$

The result is shown in Table 2. From the result, it can be seen that the predictions of trends in CFD have a small error, and thus there is an agreement with experiments under three different flow rates. Most errors are under $30 \%$ and all are under $50 \%$. The average of error is $25.21 \%$.

And then, the correlation coefficient is used to evaluate the agreement of CFD and ERT as a supplement to the relative error; it can be seen that the correlation coefficient is 0.7686 to $3.4 \mathrm{~mm}$ and 0.7647 to $5.3 \mathrm{~mm}$. As to the correlation coefficient from equation (16), a value over 0.8 means a significant correlation and the result is close to 0.8 ; thus there is certainly a similar trend between the average values from CFD and ERT. The correlation coefficient will keep the same from both normalized and original data; thus the result has a high reliability:

$$
\text { Correlation coefficient }=\frac{\sum_{i}^{n}\left(x_{i-\mathrm{CFD}}-\bar{x}_{\mathrm{CFD}}\right)\left(x_{i-\mathrm{ERT}}-\bar{x}_{\mathrm{ERT}}\right)}{\sqrt{\sum_{i=1}^{n}\left(x_{i-\mathrm{CFD}}-\bar{x}_{\mathrm{CFD}}\right)^{2}} \sqrt{\sum_{i=1}^{n}\left(x_{i-\mathrm{ERT}}-\bar{x}_{\mathrm{ERT}}\right)^{2}}}
$$

3.3. The Distribution under Different Flow Rates. The flow rate has a deep influence on the distribution of liquid in TBR; thus it is important to make a comparative analysis. In this work, the flow rate changes from $100 \mathrm{ml} / \mathrm{min}$ to $1000 \mathrm{ml} / \mathrm{min}$ and 11 different values are included in CFD. To observe the properties more comprehensively, average value and standard deviation are added to analyze the maldistribution factor. The uniformity is reflected mainly by the maldistribution factor while the trend of volume fraction is shown more clearly in mean values. In fact, these two parameters have always been used together. P4 section is chosen to study and the relationship between flow rates and mean values is shown in Figure 5. The relationship between flow rates and standard deviations is shown in Figure 6. 
TABLE 2: Comparison of normalized average values.

\begin{tabular}{|c|c|c|c|c|c|c|}
\hline \multirow{2}{*}{ Section (flow rate) } & \multicolumn{3}{|c|}{$3.4 \mathrm{~mm}$} & \multicolumn{3}{|c|}{$5.3 \mathrm{~mm}$} \\
\hline & CFD (-) & ERT (-) & Error (\%) & CFD (-) & ERT (-) & Error (\%) \\
\hline P1 $(300 \mathrm{ml} / \mathrm{min})$ & 0.25 & 0.25 & 0.67 & 0.24 & 0.22 & 11.88 \\
\hline P2 $(300 \mathrm{ml} / \mathrm{min})$ & 0.26 & 0.38 & 30.29 & 0.24 & 0.33 & 29.30 \\
\hline P3 $(300 \mathrm{ml} / \mathrm{min})$ & 0.24 & 0.44 & 45.18 & 0.24 & 0.35 & 31.66 \\
\hline $\mathrm{P} 4(300 \mathrm{ml} / \mathrm{min})$ & 0.23 & 0.45 & 48.68 & 0.23 & 0.42 & 44.75 \\
\hline $\mathrm{P} 1(700 \mathrm{ml} / \mathrm{min})$ & 0.58 & 0.44 & 24.68 & 0.65 & 0.39 & 40.53 \\
\hline $\mathrm{P} 2(700 \mathrm{ml} / \mathrm{min})$ & 0.59 & 0.65 & 9.02 & 0.63 & 0.60 & 5.26 \\
\hline P3 (700 ml/min) & 0.56 & 0.73 & 22.84 & 0.60 & 0.64 & 5.60 \\
\hline $\mathrm{P} 4(700 \mathrm{ml} / \mathrm{min})$ & 0.54 & 0.80 & 32.63 & 0.49 & 0.77 & 35.90 \\
\hline $\mathrm{P} 1(1100 \mathrm{ml} / \mathrm{min})$ & 0.94 & 0.57 & 39.57 & 0.95 & 0.52 & 44.76 \\
\hline $\mathrm{P} 2(1100 \mathrm{ml} / \mathrm{min})$ & 1 & 0.83 & 17.37 & 0.99 & 0.82 & 17.46 \\
\hline P3 $(1100 \mathrm{ml} / \mathrm{min})$ & 0.95 & 0.91 & 4.45 & 1 & 0.86 & 14.38 \\
\hline $\mathrm{P} 4(1100 \mathrm{ml} / \mathrm{min})$ & 0.86 & 1 & 13.99 & 0.90 & 1 & 9.58 \\
\hline Correlation coefficient & \multicolumn{3}{|c|}{0.7686} & \multicolumn{3}{|c|}{0.7647} \\
\hline
\end{tabular}

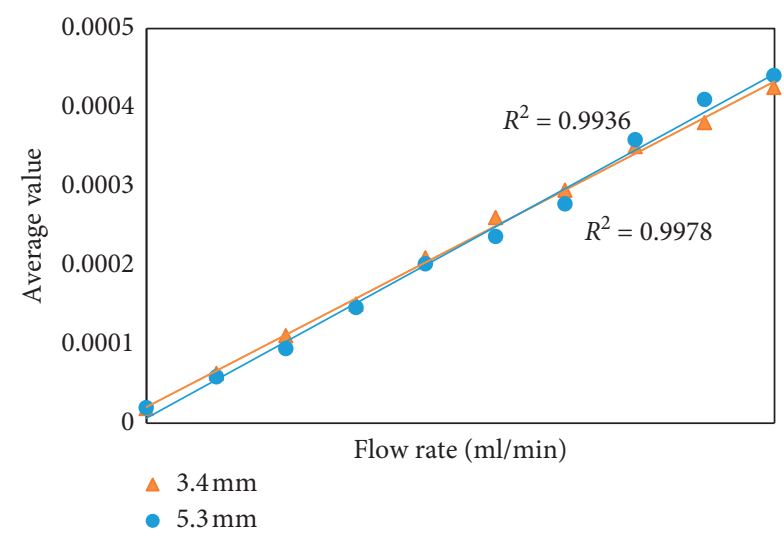

FIgURE 5: The average value with different flow rates.

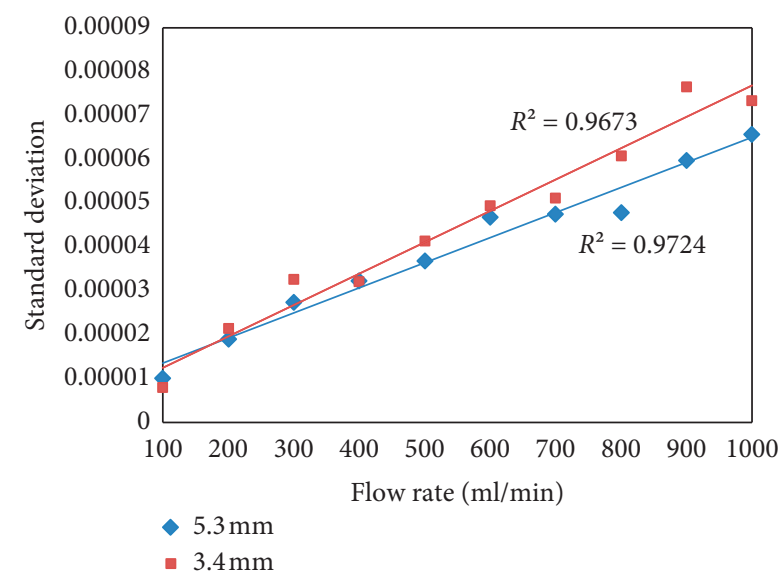

FIgURE 6: The standard deviation with different flow rates.

It can be concluded that the liquid volume fraction increases with flow rates. There are linear relationships between the mean values and standard deviations, and an exponential relationship is in the maldistribution factors for both kinds of particles. The inlet flow rates have a significant effect on the distribution of liquid phase volume fraction, which are higher than the particle diameter. Maldistribution factor keeps nearly the same in the range of $700 \mathrm{ml} / \mathrm{min}$ to
$1000 \mathrm{ml} / \mathrm{min}$ while it decreases obviously with lower flow rates.

From Figure 7, it can be observed that the liquid phase will penetrate catalyst particles well with a higher flow rate. An ideal state for TBR is that there is a higher volume fraction but a lower maldistribution factor; therefore, a higher flow rate is needed. However, the liquid holdup will also increase if the flow rate reaches a certain value, and then flooding begins seriously [23]. According to the results of P4, the maldistribution factor keeps stable in the area from $500 \mathrm{ml} / \mathrm{min}$ to $1000 \mathrm{ml} / \mathrm{min}$; meanwhile serious wall-flow phenomenon occurs when the flow rate reaches $1100 \mathrm{ml} /$ min according to Figure 3 and this should be avoided. From the result, it can be found that the flow rate of $800 \mathrm{ml} / \mathrm{min}$ is a good choice in actual work for this trickle bed reactor.

3.4. The Correlations Formula under Different Flow Rates. In order to predict the liquid volume fraction, regression predictions have been researched. The correlations formula of the relationship between the mean value and the flow rate with $5.3 \mathrm{~mm}$ particle diameter is expressed as equation (17) and with $3.4 \mathrm{~mm}$ particle diameter is expressed as equation (18). $R^{2}$ means the coefficient of determination, and it is a statistical measurement to know the approximate degree between the prediction and real data. $R^{2}$ can be calculated by equation (19).

$$
\begin{aligned}
& \bar{X}_{5.3}=5 e^{-7} v-4 e^{-5}, \\
& \bar{X}_{3.4}=5 e^{-7} v-3 e^{-5},
\end{aligned}
$$

$$
R^{2}=1-\frac{\sum_{i=1}^{n}\left(X_{i}-\hat{X}_{i}\right)^{2}}{\sum_{i=1}^{n}\left(X_{i}-\bar{X}\right)^{2}} .
$$

Here, $X_{i}$ is the observed data and $\bar{X}$ means its mean value. $\widehat{X}_{i}$ is the predicted value calculated from the coefficient of determination. If $R^{2}$ is 1 , it indicates that there is no error in prediction [24]. $R^{2}$ is 0.9936 with $5.3 \mathrm{~mm}$ and 0.9978 with $3.4 \mathrm{~mm}$ in Figure 5, respectively. The correlations formula of standard deviation with $5.3 \mathrm{~mm}$ is expressed as 


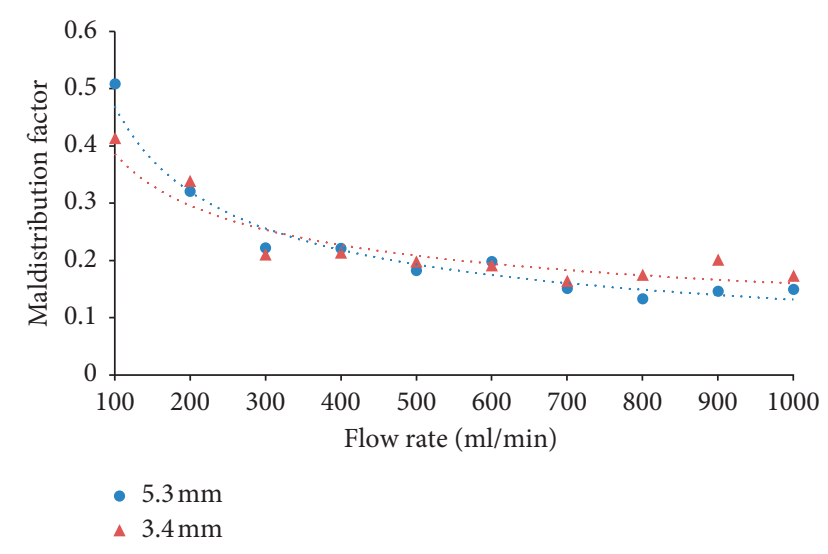

FIGURE 7: The maldistribution factor with different flow rates.

equation (20) and its $R^{2}$ is 0.9724 . The correlations formula of standard deviation with $3.4 \mathrm{~mm}$ particles is expressed as equation (21) and its $R^{2}$ is 0.9673 . The mean value and standard deviation have a good linear correlation, and the errors are within $1 \%$.

$$
\begin{aligned}
& \sigma_{5.3}=7 e^{-8} v+5 e^{-6}, \\
& \sigma_{3.4}=6 e^{-8} v+8 e^{-6} .
\end{aligned}
$$

The correlations formulas of the maldistribution factor are expressed as equations (22) and (23). Here, $R^{2}$ is 0.8539 in 5.3 particle diameter, and the other one is 0.9486 . The two correlation formulas have a good index correlation, and the errors are within $15 \%$.

$$
\begin{aligned}
& M_{f 5.3}=5.95 v^{-0.552}, \\
& M_{f 3.4}=2.25 v^{-0.382} .
\end{aligned}
$$

In order to prove the reasonability for the correlation prediction of exponential formula between $M_{f}$ and the flow rates, here $M_{f 1}$ is the value of equation (20) divided by equation (17), defined by equation (12). Similarly, $M_{f 3}$ is the value of equation (21) divided by equation (18). $M_{f 2}$ and $M_{f 4}$ represent equations (22) and (23), respectively. It can be seen from Figure 8 that the four functions have the same movement trend, and therefore the prediction formulas of $M_{f}$ on $\mathrm{P} 4$ section are reasonable. Besides, different particle diameters have little effect on the movement trend of $M_{f}$ on P4 section, while the values may be different.

Then, the intuitive analysis method is also introduced to study the weighting of influence on the flow rate from diameter more accurately. Take $S$ as the extreme deviation that reflects the influence of this factor on the mean square error of flow rate, and it is the maldistribution of average diameter and can be noted as $S_{3.4 \mathrm{~mm}}$ and $S_{5.3 \mathrm{~mm}}$ in this work. The calculation can be expressed as follows:

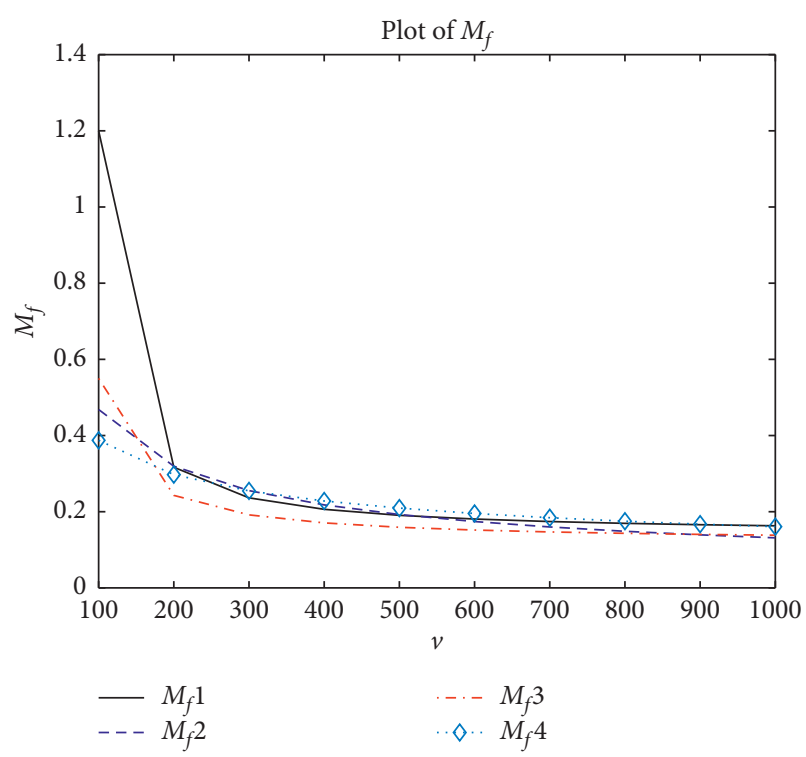

Figure 8: The relationship between flow rates and $M_{f}$.

TABLE 3: The intuitive analysis and weight of $M_{f}$.

\begin{tabular}{lcc}
\hline Flow rates $(\mathrm{ml} / \mathrm{min})$ & $M_{f}(5.3 \mathrm{~mm})$ & $M_{f}(3.4 \mathrm{~mm})$ \\
\hline 100 & 0.508 & 0.412 \\
200 & 0.320 & 0.338 \\
300 & 0.221 & 0.209 \\
400 & 0.220 & 0.212 \\
500 & 0.182 & 0.1967 \\
600 & 0.198 & 0.190 \\
700 & 0.151 & 0.163 \\
800 & 0.133 & 0.173 \\
900 & 0.146 & 0.201 \\
1000 & 0.149 & 0.172 \\
$\mathrm{~S}$ & 0.495 & 0.505 \\
\hline
\end{tabular}

$$
\begin{aligned}
& S_{3.4 \mathrm{~mm}}=\frac{M_{f 3.4}}{M_{f 3.4}+M_{f 5.3}}, \\
& S_{5.3 \mathrm{~mm}}=\frac{M_{f 5.3}}{M_{f 3.4}+M_{f 5.3}} .
\end{aligned}
$$

$S$ can be used as the coefficient to describe the weighting of two diameters and obtained by equations (22) and (23) under different flow rates. These two factors will compose the prediction model as coefficients. Results are shown in Table 3. Finally, the maldistribution of P4 section with known inlet flow rate $F$ can be predicted by equation (26) with $S$.

$$
F=M_{f 5.3} \times S_{5.3 \mathrm{~mm}}+M_{f 3.4} \times S_{3.4 \mathrm{~mm}}=2.95 v^{-0.552}+1.14 v^{-0.382} .
$$


TRB always works under high temperature and pressure and it is almost impossible to directly observe the flow state of internal liquid phase from the outside. Thus, the given prediction model has a significance for the design and optimization in TBR. In addition, results in this part may be effective ranging within $300-1100 \mathrm{ml} / \mathrm{min}$ in experiment and $100-1100 \mathrm{ml} / \mathrm{min}$ in CFD.

\section{Conclusion}

In order to find the distribution and property of liquid phase in TBR, a series of researches are taken based on both CFD and ERT. The distribution of liquid phase was studied with particles of different average diameters and a series of flow rates. The maldistribution factor is used to evaluate the impact factor in trickle bed reactor. Then, the volume fractions of liquid phase have been predicted with CFD. The main conclusions are as follows:

(1) The distribution of multiple phases was simulated for a TBR with a mixed model based on CFD. A laminar flow part is used to describe the particle filling area and a turbulent model for the rest. A series of parameters in CFD is calculated and chosen. Boundary conditions are set based on the ERT experiment in [16]. The distribution of flow volume fractions was obtained effectively. In order to compare the results from different fields, a dimensionless unit, maldistribution factor, was used.

(2) The visualization for CFD models was also made to describe the distribution of liquid phase, and images in sections were obtained. The degree of dispersion for distribution is described by the maldistribution factor in different sections and it is found that the liquid is relatively uneven near the inlet and then is blocked by the particles. A clearer process can be observed directly and the effect of maldistribution is also proved. The maldistribution of $5.3 \mathrm{~mm}$ is higher than $3.4 \mathrm{~mm}$, and trends of maldistributions predicted in CFD were in an agreement with experimental results. Besides, wall-flow also can be found and it will be more obvious with a higher flow rate.

(3) Average values were normalized with a min-max normalization and then compared between CFD and ERT. From the result, it can be seen that the predictions of trends in CFD have an average error of $25.21 \%$, and thus there is an agreement with experiments under three different flow rates. From the correlation coefficient, there is certainly a similar trend between the average values from CFD and ERT. The distribution under different flow rates was studied. The liquid volume fraction increases with flow rates. There are linear relationships between the mean values and standard deviations, and an exponential relationship is in the maldistribution factors. Maldistribution factor keeps nearly the same in the range of $700 \mathrm{ml} / \mathrm{min}$ to $1000 \mathrm{ml} / \mathrm{min}$ while it decreases obviously with lower flow rates.
(4) The correlation formulas under different flow rates were analyzed. In order to predict the liquid volume fraction, regression predictions have been researched. The correlation formulas of the relationship between the mean value and the flow rate were calculated, and the results were evaluated. The mean value and standard deviation have a good linear correlation, and the errors are within $1 \%$. Then, the intuitive analysis method is also introduced to study the weighting of influence on the flow rate from diameter more accurately. The weighting of two diameters was described under different flow rates. These two factors will compose the prediction model as coefficients, therefore, a more specific formula to predict flow rate with different maldistributions.

The obtained regression model can be used for the design of the trickle bed reactor and the evaluation for properties of the internal liquid phase. In addition, this work established a reasonable model to get the properties of TBR based on CFD method, and then most steps and conclusions can be used to save cost in experiment and design of TBR. Since there are many different conditions from ideal state, this paper mainly focuses on the verification of CFD model, and most experiments are finished before.

\section{Data Availability}

The data used to support the findings of this study are true and reliable and are available from the corresponding author upon request.

\section{Conflicts of Interest}

The authors declare that they have no conflicts of interest.

\section{Acknowledgments}

This work was supported by the National Natural Science Foundation of China (Grant 51876175) and the Technology Innovative Leading Program of Shaanxi (Grant 2019 CGXNG-030). The authors wish to thank the reviewers for their advice and opinions as they were very important and useful.

\section{References}

[1] A. Arnab, R. Shantanu, and L. Faïçal, "Cyclic operation of trickle bed reactors: a review," Chemical Engineering Science, vol. 115, pp. 205-214, 2014.

[2] D. I. A. Dhanraj and V. V. Buwa, "Effect of capillary pressure force on local liquid distribution in a trickle bed," Chemical Engineering Science, vol. 191, pp. 115-133, 2018.

[3] L. Katja, M. Mikko, and A. Ville, "CFD modeling of radial spreading of flow in trickle-bed reactors due to mechanical and capillary dispersion," Chemical Engineering Science, vol. 64, pp. 207-218, 2009.

[4] M. Bazmi, S. H. Hashemabadi, and M. Bayat, "CFD simulation and experimental study of liquid flow mal-distribution through the randomly trickle bed reactors," International 
Communications in Heat and Mass Transfer, vol. 39, no. 5, pp. 736-743, 2012.

[5] A. H. Beni and M. R. Khosravi-Nikou, "Modeling hydrodynamics of trickle-bed reactors at high and low pressure using CFD method," Petroleum Science and Technology, vol. 33, no. 20, pp. 1770-1779, 2015.

[6] K. R. Rupesh and B. J. Jyeshtharaj, "CFD modeling of pressure drop and drag coefficient in fixed beds: wall effects," Particuology, vol. 8, no. 1, pp. 37-43, 2010.

[7] P. Mohammad, Z. Mohammadjavad, and G. Poobalan, "Prediction of multiphase flow pattern inside a 3D bubble column reactor using a combination of CFD and ANFIS," RSC Advances, vol. 104, p. 1039, 2015.

[8] M. D. Amir and L. Faical, "CFD study and experi-mental validation of multiphase packed bed hydrodynamics in the context of rolling sea conditions," AIChE Journal, vol. 65, pp. 385-397, 2019.

[9] T. Schäfer, C. Meitzner, R. Lange, and U. Hampel, "A study of two-phase flow in monoliths using ultrafast single-slice X-ray computed tomography," International Journal of Multiphase Flow, vol. 86, pp. 56-66, 2016.

[10] B. Christophe, A. Koudil, P. Chen, and M. P. Dudu-kovic, "Study of liquid spreading from a point source in a trickle bed via gamma-ray tomography and CFD simulation," Chemical Engineering Science, vol. 60, pp. 6279-6288, 2005.

[11] L. F. Gladden, M. H. M. Lim, M. D. Mantle, A. J. Sederman, and E. H. Stitt, "MRI visualisation of two-phase flow in structured supports and trickle-bed reactors," Catalysis Today, vol. 80, no. 30, pp. 203-210, 2003.

[12] Y. Fuhe, A. Artin, and K. Nandakumar, "Liquid holdup distribution in packed columns: gamma ray tomography and CFD simulation," Chemical Engineering and Processing, vol. 41, pp. 473-483, 2002.

[13] A. Wang, Q. Marashdeh, B. J. Motil, and L.-S. Fan, "Electrical capacitance volume tomography for imaging of pulsating flows in a trickle bed," Chemical Engineering Science, vol. 119, pp. 77-87, 2014.

[14] R. L. Mcmanus, G. A. Funk, M. P. Harold, and K. M. Ng, "Experimental study of reaction in trickle-bed reactors with liquid maldistribution," Industrial \& Engineering Chemistry Research, vol. 32, no. 3, pp. 570-574, 1993.

[15] T. Eda, A. Sapkota, and M. Takei, "Investigation of liquid dispersion in the packed-bed by electrical resistance tomography," in Proceedings of the International Conference on Nuclear Engineering \& the Asme Power Conference, pp. 447450, Anaheim, CA, USA, July 2012.

[16] T. Eda, A. Sapkota, J. Haruta, M. Nishio, and M. Takei, "Experimental study on liquid spread and maldistribution in the trickle bed reactor using electrical resistance tomography," Journal of Power and Energy Systems, vol. 7, no. 2, pp. 94-105, 2013.

[17] T. Zhao, T. Eda, S. Achyut, J. Haruta, M. Nishio, and M. Takei, "Investigation of pulsing flow regime transition and pulse characteristics in trickle-bed reactor by electrical resistance tomography," Chemical Engineering Science, vol. 130, pp. 8$17,2015$.

[18] A. Arnab, R. Shantanu, and K. D. P. Nigam, "Prediction of pressure drop and liquid holdup in trickle bed reactor using relative permeability concept in CFD," Chemical Engineering Science, vol. 62, pp. 5870-5879, 2007.

[19] A. Heidari and S. H. Hashemabadi, "CFD study of diesel oil hydrotreating process in the non-isothermal trickle bed reactor," Chemical Engineering Research and Design, vol. 94, pp. 549-564, 2015.
[20] J. G. L. Rodrigo and M. Q. Rosa, "CFD modelling of multiphase flow distribution in trickle beds," Chemical Engineering Journal, vol. 147, pp. 342-355, 2009.

[21] J. G. L. Rodrigo and M. Q. Rosa, "Turbulence modelling of multiphase flow in high-pressure trickle-bed reactors," Chemical Engineering Science, vol. 64, pp. 1806-1819, 2009.

[22] Z. Ren, A. Kowalski, and T. L. Rodgers, "Measuring inline velocity profile of shampoo by electrical resistance tomography (ERT)," Flow Measurement and Instrumentation, vol. 58, pp. 31-37, 2017.

[23] E. Hansuld, L. Briens, and C. Briens, "Acoustic detection of flooding in absorption columns and trickle beds," Chemical Engineering and Processing: Process Intensification, vol. 47, no. 5, pp. 871-878, 2008.

[24] Z. M. Yaseen, S. O. Sulaiman, R. C. Deo, and K.-W. Chau, "An enhanced extreme learning machine model for river flow forecasting: state-of-the-art, practical applications in water resource engineering area and future research direction," Journal of Hydrology, vol. 569, pp. 387-408, 2019. 